\title{
4th National Anti-tuberculosis Drug Resistance Survey
} \section{in Kenya}

Joseph Sitienei $^{1}$, Kamene Kimenye ${ }^{1}$, Josephine Wahogo ${ }^{1,6}$, Bernard Langat ${ }^{1}$, Enos Masini ${ }^{1}$, Obadiah Njuguna ${ }^{1}$, Jane Ong'ang'o ${ }^{2}$, Josephine Wahogo ${ }^{3}$, Sophie Matu ${ }^{3}$, Jeremiah Okari ${ }^{3}$, Maurice Maina ${ }^{4}$, Margret Mburu ${ }^{5}$, Herman Weyenga $^{5}$, Jane Mwangi ${ }^{5}$, Lucy Nganga ${ }^{5}$, Agnes Langat ${ }^{5}$, Abraham Katana ${ }^{5}$, Hillary Kipruto ${ }^{1,6}$ and Joel Kangangi ${ }^{6}$

1. Division of Leprosy Tuberculosis and Lung Disease, Department of Disease Prevention and Control, Ministry of Health, Nairobi 20781, Kenya

2. Kenya Medical Research Institute [KEMRI], Centre for Respiratory Diseases Research, Nairobi 20781, Kenya

3. National TB Reference Laboratory, Department of Disease Prevention and Control, Ministry of Health, Nairobi 20781, Kenya

4. United States Agency for International Development [USAID], Department of Population and Health, Kenya and East Africa Office, Nairobi 20781, Kenya

5. U.S. Centers for Disease Control and Prevention [CDC], Nairobi 20781, Kenya

6. World Health Organization [WHO], Kenya Country Office, Nairobi 20781, Kenya

\begin{abstract}
Introduction: Recently rapid development of drug resistant TB, particularly MDR TB (Multi Drug Resistant TB) and XDRTB (Extensively Drug-Resistant TB) possess a major threat to control of tuberculosis globally. Information on the extent of MDR-TB from Kenya is largely limited due to several factors. Monitoring of development of resistance is a vital tool in providing critical information for effective planning for TB control and in management of patients infected with TB. Methods: Cross-sectional with cluster design. Results: A total of 2,171 participants recruited into the study from 50 selected clusters. Prevalence of rifampicin resistance for new cases was 1.3\% [95\% CI, 0.8-2.0] and INH resistance was 5.5\% [95\% CI, 4.5-6.7]. MDR TB was found in 0.67\% of new cases and $2.1 \%$ amongst previously treated TB cases. Discussion: Resistance to isoniazid in Kenya has been on the decline due to introduction of rifampicin in combined therapy. There was increase of MDR TB among new cases by $24 \%$ and decline in previously treated cases due to lethal impact of HIV. Conclusions: Although drug resistance TB is a growing problem in Kenya, resistance to isoniazid and rifampicin MDR TB is less than previously estimated. The country should continue to monitor drug resistance and ensure effective use of anti TB medicines.
\end{abstract}

Key words: Multi drug resistant TB, Mycobacterium tuberculosis, drug resistance, Kenya.

\section{Introduction}

Recently rapid development of drug resistant TB, particularly MDR TB (Multi Drug Resistant TB) and XDR TB (Extensively Drug-Resistant TB) possess a major threat to the control of tuberculosis globally [1] and threatens to reverse gains made in global TB control. The WHO (World Health Organization) has set ambitious targets to be met if the world is to end the TB epidemic by 2035 [2]. Information on the

Corresponding author: Dr. Joseph Sitienei, Ph.D, research fields: TB and lung disease. extent of MDR TB from African region is largely limited due to several factors including poor laboratory infrastructure, poor surveillance mechanisms and reporting procedures, out dated databases and sub-optimal coverage of infrequent surveys [3].

Monitoring development of drug resistance is a vital tool in providing critical information for effective planning for $\mathrm{TB}$ control and in management of patients infected with TB [4]. WHO recommends countries to conduct periodic surveillance of drug resistance to monitor the trends of resistance over time. In recognition of the need for strengthening global 
surveillance for drug-resistant TB, the 2009 World Health Assembly resolution WHA62.15 reiterated "Prevention and control of multidrug-resistant tuberculosis and extensively drug-resistant tuberculosis" and urged all Member States to "achieve universal access to diagnosis and treatment of multidrug-resistant and extensively drug-resistant tuberculosis”.

Drug Resistant TB is caused by Mycobacterium tuberculosis that is resistant to any one of the four main anti-TB medicines. It poses a huge challenge for many countries and in particular to countries with a high HIV burden. Most of these countries are in SSA (Sub Sahara Africa), which houses the highest burden of the dual epidemic [3]. Diagnosis and management of resistant TB are not only expensive, but also very challenging to countries with limited resources [5].

Updated WHO guidelines for surveillance of drug resistance in tuberculosis incorporate experience gained from 20 years of the Global Project on Anti-tuberculosis Drug Resistance Surveillance Initiated by $\mathrm{WHO}$ and the International Union against Tuberculosis and Lung Disease [6]. Kenya participated in the global drug resistance survey of 1994 and has conducted several surveys limited to a few parts of the country. The results from these surveys have not been very conclusive and over the years, the country has relied on estimates to plan for TB control services.

These local surveys have identified a combination of several risk factors that lead to development of drug resistance. These factors are similar to what has been documented elsewhere and include prior exposure to TB medicines, poor adherence to medication and poor quality of medicines and HIV infection amongst others. These factors are all relevant to the Kenyan context. In countries with limited resources, the combination of drug resistant TB, HIV and limited to access to therapeutic regimens of first and second-line medicines has catastrophic outcomes with high mortality rates $[7,8]$.
For Kenya to develop a comprehensive national response to drug-resistant tuberculosis and to achieve global targets, the NLTD-P (National Leprosy, TB and Lung Disease Program) conducted a countrywide national surveillance survey of drug-resistant tuberculosis. The objectives of the survey were to determine the proportion of tuberculosis cases resistant to TB drugs, estimate the incidence of drug-resistant tuberculosis, and identify the factors that are linked to drug-resistant tuberculosis, especially to MDR tuberculosis.

\section{Methods}

The cross-sectional survey used cluster design as recommended by the WHO in the guidelines for surveillance of TB drug resistance (WHO, 2015). Using the existing 290 districts in the country as clusters, 50 clusters were randomly selected and the calculated sample size was allocated to each cluster (district) equally, within each cluster the samples were allocated to health facilities proportionate to the number of smear positive TB cases reported to the National TB Control program the previous year (2013) be each of the health facilities. The calculated proportion of cases was also distributed proportionately to the diagnostic health facilities in the each cluster.

All newly diagnosed smear positive TB cases and previously treated cases in the cluster registered during the study period of 1st April 2014 and 30th March 2015 and who consented were enrolled into the study until the allocated sample size for the facility was reached.

The sample size calculation was based on an expected prevalence of MDR TB of $1-2 \%$ in new smear positive TB cases. The crude sample size was doubled to accommodate an estimated design effect of 2. The sample size was increased by $20 \%$ to accommodate for.

A sample size of 2,500 was reached using SRS (simple random sampling), with finite population correction. 


\subsection{Field Procedures}

\section{Participants' Recruitment}

The survey was conducted within the routine set up of diagnosing TB patients in the health care facilities. All new smear positive TB participants and all smear positive re-treatment cases seeking care in the health facilities during the study period were eligible for inclusion. Those eligible and fulfilling the inclusion criteria were requested to participate in the survey by providing them information related to the study. Those who consented were asked to sign a consent form which had been translated to the local language-Kiswahili. This was done before a sputum sample that was collected from the participant. For all eligible cases under 18 years, assent was sought from guardians.

Each participant was assigned a unique pre-printed bar code identification number and registered in the Facility DRS (Drug Resistant Survey) study register. The bar code label was also attached on all survey forms, specimen containers and facility DRS study register. If for any reason, no sample was obtained and submitted to NTRL (National TB Reference Laboratory), the reason for this was indicated as a remark in the facility DRS study register.

\subsection{Sputum Specimen Collection}

Participants were instructed on how to collect sputum specimen in order to get quality specimen and not saliva. To prevent disease transmission, sputum collection took place in a designated well-ventilated area and not within the laboratory. A spot specimen sample was collected from all eligible participants using a $50 \mathrm{~mL}$ screw-cap falcon tube container (Falcon, Becton Dickenson, USA). These containers were labelled using already generated bar codes and packaged by a trained technologist and sent to NTRL for culture and DST (Drug Susceptibility Testing). Samples collected and not transported on the same day were stored at $4{ }^{\circ} \mathrm{C}$ while awaiting transportation. The specimens were transported within 24 hours of collection in a cooler box through a courier system and temperatures recorded on arrival at the NTRL. The CRF (Case Recording Form) and sputum shipment forms dully filled with participant details were sent alongside the samples to the NTRL.

\subsection{Specimen Management at NTRL}

At NTRL, specimens were unpackaged from the specimen transport boxes in a dedicated biological safety cabinet and checked for leakage. Verification of the bar code on the sample was done to ensure that it matched the bar code number on the CRF. The CRF was checked for completeness and if any information was missing, the facility that sent the sample was contacted to obtain the missing information immediately. The bar code was scanned and data from the CRF entered into the LIS (laboratory information system). A laboratory register dedicated to the DRS served as paper back up of the electronic data.

Specimen was processed in the laboratory according to study protocol for ZN (Ziehl Neelsen) stain, liquid and solid culture [details available]. Physical identification of positive cultures for MTB (Mycobacteria tuberculosis) complex was done on culture plates. Species identification was done using capilia test kit and SD Bioline TB Ag MPT64. All culture positive specimen were subjected to MDR TB plus kit HAIN LifeScience according to manufacturer's instructions to confirm if they were TB samples and check for INH and rifampicin resistance. In case of discordant DST results, LPA (Line Probe Assay) was taken as the final determining result as it was the only genotypic method at the NTRL. Where MOTT/NTM was identified, further speciation was done using all species mycobacterium kit/common mycobacterium kit. LJ (Lowenstein Jensen media) was considered as the gold standard for DST. In case of a discrepancy between the phenotypic and genotypic methods, the LJ results were considered as the tie breaker and used as the final results. An 
equal volume of positive isolates were mixed with equal volume of MGIT 960 media and aliquoted into a cryo-vial and stored at $-80^{\circ} \mathrm{C}$.

Results for any identified MDR TB or rifampicin resistance was relayed through email and hard copy to the CTLC/SCTLC and the clinician for patients' management. All efforts were made by NTLD-P to ensure that cases were initiated on treatment according to existing national guidelines.

The NTRL is linked to a SRL (Supra-National Laboratory) in Brisbane, Australia which ensured that quality [Internal and External] in all processes in the laboratory were upheld. For external quality assurance of the DST, samples of positive cultures were sent to the SRL. Several technical assistance was provided by the SRL during the study period.

\subsection{Data Analysis}

Data collected were analyzed using descriptive statistics, univariate and multivariate logistic regression analyses were performed to determine if baseline characteristics of patients are associated with any drug resistance. OR (odds ratio) and 95\% CI was calculated to measure the degree of association between variables.

\subsection{Ethical Considerations}

The study protocol was approved and ethically cleared by ethical and scientific committee at KEMRI (Kenya Medical Research Institute). Each participant who met the eligibility criteria consented after being given information about the survey.

\section{Results}

A total of 2,171 participants (86.8\% estimated sample size) were recruited into the study from 50 selected clusters from April 2014 to March 2015. More than half (55.8\%) of the study participants were within the economically productive age group of 25-44 years. Males constituted $64.8 \%$ of all the study participants, 63.8\% $(1,219)$ among new and $72.5 \%$
(187) among previously treated TB cases. More than $43.7 \%$ (949) of the participants were married while $44.1 \%$ (969) were single. Majority of the participants, $73.1 \%$ had received some form of formal education (primary, secondary or tertiary). Participants in employment (both formal and informal) constituted $69.2 \%(1,503)$ while $11.3 \%$ were students. Cigarette smoking was reported among $21.7 \%$ of study participants while $48 \%$ of study participants reported alcohol consumption as shown in Table 1.

About 20.6\% (448) participants were HIV infected. Amongst the new TB cases, $19.2 \%$ were HIV infected while amongst previously treated TB cases, $31.8 \%$ were HIV infected. Almost $2 \%$ of the participants were diabetics and about 5\% asthmatics. More than $28.7 \%$ of study participants reported a history of contact with TB while $18.8 \%$ had a household contact.

Fig. 1 shows the flow of participants and specimen in the survey. Participants included 1,910 new cases, 258 previously treated cases and 3 cases with unknown treatment history. Culture results were available for 2,168 specimens from 1,907 new and 258 previously treated cases. Culture of specimen yielded 10 cases of non tuberculous TB (four of these were HIV negative, five HIV positive and one unknown HIV status) and 1,845 MTB cases. A total of 123 specimens were contaminated while 190 were culture negative. Of the 1,845 specimen that were culture positive, 1,842 specimens had DST results. A total of 15 MDR TB cases were identified from the specimen submitted to the NTRL.

Table 2 shows the different resistance patterns at the end of the survey for new and previously treated TB cases. Resistance to amongst new TB cases for rifampicin, isoniazid and ethambutol was 1.3\%, 5.2\% and $3.1 \%$ respectively. As expected, higher resistance was noted among previously treated patients than new cases. The levels of resistance for previously treated cases were $5.2 \%, 6.8 \%, 4.1 \%$ for rifampicin, isoniazid and ethambutol respectively. Resistance to streptomycin was $6.3 \%$ for new and previously treated 
cases. Mono resistance for new TB cases remained below $5 \%$ for the three drugs (rifampicin, isoniazid and ethambutol) with the highest reported for ethambutol at $4.4 \%$, while for previously treated TB patients, monoresistance was $2.6 \%$ for all the medicines.

Table 1 Participants' socio demographic characteristics.

\begin{tabular}{|c|c|c|c|c|c|c|c|c|}
\hline \multirow{3}{*}{$\begin{array}{l}\text { Variable } \\
\text { Gender }\end{array}$} & \multicolumn{8}{|c|}{ Treatment history } \\
\hline & \multicolumn{2}{|c|}{ New cases } & \multicolumn{2}{|c|}{ Previously treated } & \multicolumn{2}{|c|}{ Missing } & \multicolumn{2}{|c|}{ All cases } \\
\hline & No. & $\%$ & No. & $\%$ & No. & $\%$ & No. & $\%$ \\
\hline Male & 1,219 & 63.8 & 187 & 72.5 & 1 & 33.3 & 1,407 & 64.8 \\
\hline Female & 689 & 36.1 & 71 & 27.5 & 2 & 66.7 & 762 & 35.1 \\
\hline Missing & 2 & 0.1 & 0 & 0 & 0 & 0 & 2 & 0.1 \\
\hline \multicolumn{9}{|l|}{ Age groups (years) } \\
\hline $0-14$ & 44 & 2.3 & 3 & 1.2 & 0 & 0 & 47 & 2.2 \\
\hline $15-24$ & 480 & 25.1 & 36 & 14 & 0 & 0 & 516 & 23.8 \\
\hline $25-34$ & 675 & 35.3 & 98 & 38 & 2 & 66.7 & 775 & 35.7 \\
\hline $35-44$ & 365 & 19.1 & 71 & 27.5 & 0 & 0 & 436 & 20.1 \\
\hline $45-54$ & 162 & 8.5 & 33 & 12.8 & 0 & 0 & 195 & 9.0 \\
\hline $55-64$ & 85 & 4.5 & 10 & 3.9 & 1 & 33.3 & 96 & 4.4 \\
\hline $65+$ & 38 & 2.0 & 4 & 1.6 & 0 & 0 & 42 & 1.9 \\
\hline Missing & 61 & 3.2 & 3 & 1.2 & 0 & 0 & 64 & 2.9 \\
\hline \multicolumn{9}{|l|}{ Marital status } \\
\hline Married & 841 & 44.0 & 107 & 41.5 & 1 & 33.3 & 949 & 43.7 \\
\hline Divorced & 31 & 1.6 & 11 & 4.3 & 0 & 0 & 42 & 1.9 \\
\hline Separated & 102 & 5.3 & 19 & 7.4 & 0 & 0 & 121 & 5.6 \\
\hline Single & 857 & 44.9 & 111 & 43 & 1 & 33.3 & 969 & 44.6 \\
\hline Widower & 66 & 3.5 & 9 & 3.5 & 1 & 33.3 & 76 & 3.5 \\
\hline Missing & 13 & 0.7 & 1 & 0.4 & 0 & 0 & 14 & 0.6 \\
\hline \multicolumn{9}{|l|}{ Education } \\
\hline Primary & 834 & 43.7 & 116 & 45 & 1 & 33.3 & 951 & 43.8 \\
\hline Secondary & 405 & 21.2 & 44 & 17.1 & 0 & 0 & 449 & 20.7 \\
\hline Tertiary & 171 & 9.0 & 15 & 5.8 & 1 & 33.3 & 187 & 8.6 \\
\hline No formal education & 451 & 23.6 & 73 & 28.3 & 1 & 33.3 & 525 & 24.2 \\
\hline Other & 41 & 2.1 & 7 & 2.7 & 0 & 0 & 48 & 2.2 \\
\hline Missing & 8 & 0.4 & 3 & 1.2 & 0 & 0 & 11 & 0.5 \\
\hline \multicolumn{9}{|l|}{ Occupation } \\
\hline Employed & 283 & 14.8 & 48 & 18.6 & 0 & 0 & 331 & 15.2 \\
\hline Self employed & 674 & 35.3 & 87 & 33.7 & 1 & 33.3 & 762 & 35.1 \\
\hline Informal employment & 357 & 18.7 & 52 & 20.2 & 1 & 33.3 & 410 & 18.9 \\
\hline Unemployed & 331 & 17.3 & 49 & 19 & 1 & 33.3 & 381 & 17.5 \\
\hline Student & 229 & 12.0 & 17 & 6.6 & 0 & 0 & 246 & 11.3 \\
\hline Other & 31 & 1.6 & 5 & 1.9 & 0 & 0 & 36 & 1.7 \\
\hline Missing & 5 & 0.3 & 0 & 0 & 0 & 0 & 5 & 0.2 \\
\hline \multicolumn{9}{|l|}{ Cigarette smoking } \\
\hline Smoking & 414 & 21.7 & 62 & 24 & 0 & 0 & 476 & 21.9 \\
\hline Non-smoking & 1,176 & 61.6 & 160 & 62 & 1 & 33.3 & 1,337 & 61.6 \\
\hline Missing & 320 & 16.8 & 36 & 14 & 2 & 66.7 & 358 & 16.5 \\
\hline \multicolumn{9}{|l|}{ Alcohol use } \\
\hline Use alcohol & 887 & 46.4 & 153 & 59.3 & 1 & 33.3 & 1,041 & 48.0 \\
\hline Do not use alcohol & 988 & 51.7 & 103 & 39.9 & 2 & 66.7 & 1,093 & 50.3 \\
\hline Missing & 35 & 1.8 & 2 & 0.8 & 0 & 0 & 37 & 1.7 \\
\hline
\end{tabular}




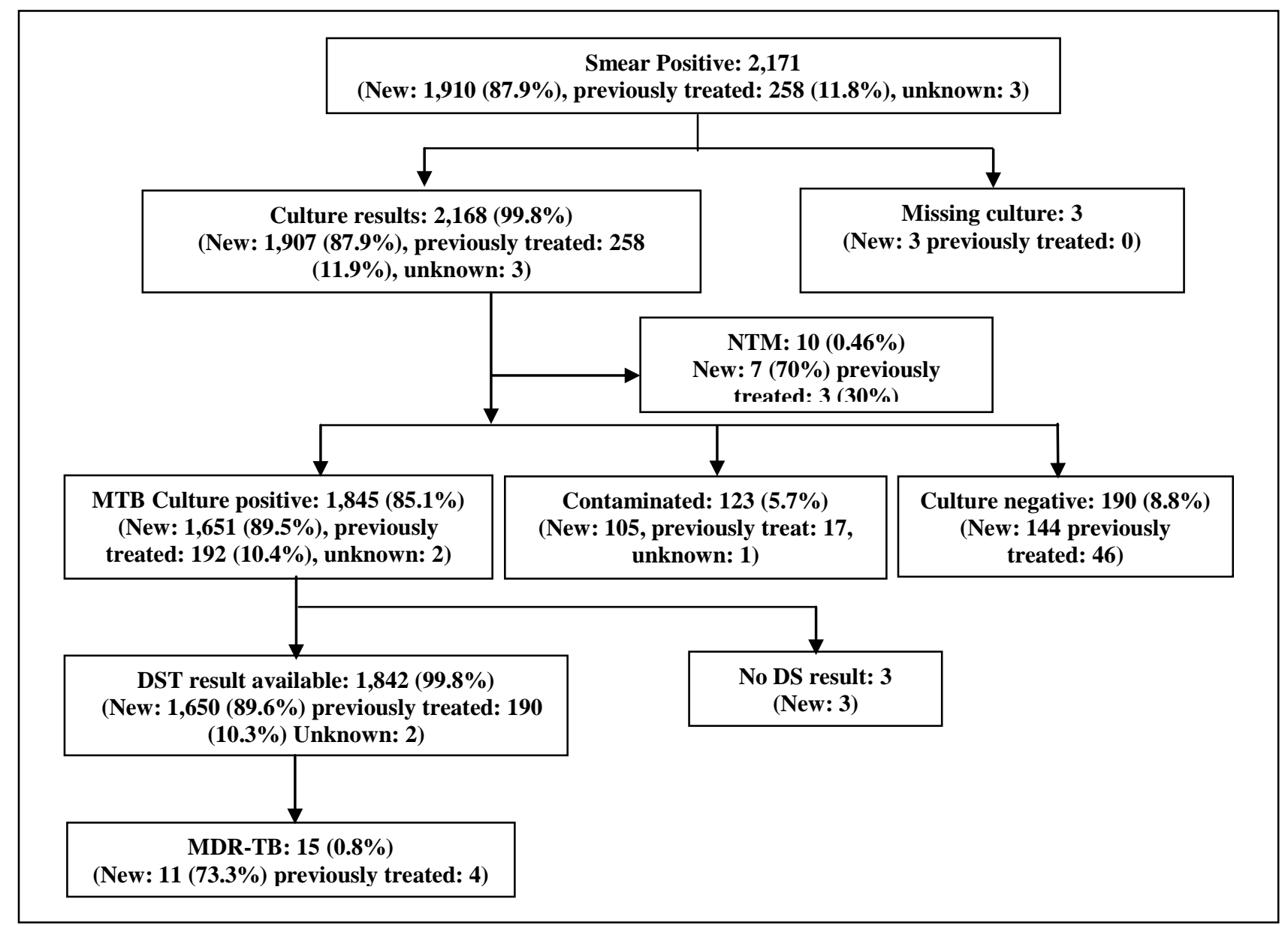

Fig. 1 Cascade of specimen.

Table 2 Prevalence and patterns of resistance to first line anti-TB drugs in Kenya.

\begin{tabular}{|c|c|c|c|c|c|c|c|c|c|c|}
\hline \multirow[b]{2}{*}{ Drug resisntcence } & & \multirow[b]{2}{*}{$\mathrm{N}$} & \multicolumn{2}{|c|}{ New cases } & \multicolumn{3}{|c|}{ Previously treated cases } & \multicolumn{2}{|c|}{ All cases } & \multirow[b]{2}{*}{$95 \% \mathrm{CI}$} \\
\hline & & & $\%$ & $95 \% \mathrm{CI}$ & $\mathrm{N}$ & $\%$ & $95 \% \mathrm{CI}$ & $\mathrm{N}$ & $\%$ & \\
\hline \multirow{5}{*}{ Any resistance } & Rifampicin (R) & 22 & 1.3 & $(0.8-2.0)$ & 10 & 5.2 & $(2.5-9.4)$ & 32 & 1.7 & $(1.2-2.4)$ \\
\hline & Isoniazid (H) & 85 & 5.2 & $(4.1-6.3)$ & 13 & 6.8 & $(3.7-11.3)$ & 98 & 5.3 & $(4.3-6.4)$ \\
\hline & Ethambutal (E) & 52 & 3.1 & $(2.3-4.1)$ & 8 & 4.1 & $(1.8-8.0)$ & 60 & 3.3 & $(2.5-4.2)$ \\
\hline & Streptomycin (S) & 101 & 6.3 & $(5.1-7.5)$ & 12 & 6.3 & $(3.2-10.7)$ & 113 & 6.3 & $(5.1-7.3)$ \\
\hline & Rifampicin (R) & 8 & 0.5 & $(0.2-1.0)$ & 5 & 2.6 & $(0.8-6.0)$ & 13 & 0.7 & $(0.3-1.2)$ \\
\hline \multirow{3}{*}{ Mono resistance } & Isoniazid (H) & 49 & 4.2 & $(3.3-5.4)$ & 5 & 2.6 & $(0.8-6.0)$ & 54 & 2.9 & $(2.2-3.8)$ \\
\hline & Ethambutal (E) & 71 & 4.4 & $(3.5-5.5)$ & 5 & 2.6 & $(0.8-6.0)$ & 76 & 4.1 & $(3.2-5.1)$ \\
\hline & Streptomycin (S) & 27 & 1.7 & $(1.1-2.4)$ & 5 & 2.6 & $(0.8-6.0)$ & 32 & 1.7 & $(1.2-2.4)$ \\
\hline \multirow{7}{*}{$\begin{array}{l}\text { PDR (poly drug } \\
\text { resistant) }\end{array}$} & RS & 0 & - & - & 0 & - & - & 0 & - & - \\
\hline & $\mathrm{RE}$ & 3 & 0.2 & $(0.04-0.5)$ & 1 & 0.5 & $(0.12-2.8)$ & 4 & 0.22 & $(0.06-0.55)$ \\
\hline & $\mathrm{HE}$ & 10 & 0.6 & $(0.29-1.11)$ & 3 & 1.5 & $(0.3-4.4)$ & 13 & 0.7 & $(0.38-1.2)$ \\
\hline & HS & 7 & 0.4 & $(0.2-0.8)$ & 0 & - & - & 7 & 0.38 & $0.15-0.78$ \\
\hline & ES & 3 & 0.2 & $(0.04-0.5)$ & 0 & - & - & 3 & 0.16 & $(0.03-0.47)$ \\
\hline & RES & 0 & - & - & 0 & - & - & 0 & - & - \\
\hline & HES & 8 & 0.5 & $(0.2-0.95)$ & 1 & 0.5 & $(0.12-2.8)$ & 9 & 0.49 & $(0.22-0.92)$ \\
\hline \multirow{3}{*}{$\begin{array}{l}\text { MDR (multi } \\
\text { drug resistant) }\end{array}$} & $\mathrm{RH}$ & 4 & 0.24 & $0.07-0.62$ & 2 & 1.0 & $(0.13-3.71)$ & 6 & - & - \\
\hline & RHS & 1 & 0.1 & $(0.002-0.34)$ & 0 & & & 1 & 0.05 & $(0.001-0.30)$ \\
\hline & RHES & 6 & 0.4 & $(0.13-0.79)$ & 2 & 1.0 & $(0.13-3.71)$ & 8 & 0.45 & $(0.19-0.85)$ \\
\hline
\end{tabular}


Combination of isoniazid and ethambutol had the highest poly resistance at $0.6 \%$ for new TB cases while for previously treated cases, this increased to 1.5\%. Poly resistance for HES (Isoniazid, Ethambutol and Streptomycin) combination was about 5\% for new and previously treated cases.

There were 1,313 (71.3\%) cases who were pan sensitive to all first line medicines used to treat TB while 532 (28.7\%) had some form of drug resistance to either single or multiple drugs. Standard logistic regression indicated MDR prevalence of 0.67 (95\% CI; $0.2-1.1$ ) among the new cases and for previously treated cases a prevalence of 2.0 (95\% CI; 0.2-3.9). Multi drug resistant TB was detected in 15 study participants, six who were HIV infected. One case of MDR TB was detected from amongst 35 Diabetics and one case from 107 Asthmatics.

\section{Discussions}

This survey aimed at finding the TB drug resistance situation in Kenya in 2015. The results are particularly important for the country to build additional information on resistance patterns for conventionally used medicines. The findings of the survey build on what was found in the Global Surveillance that Kenya participated in 2012 [9] and other previous limited and national surveys. This survey has revealed low levels of drug resistance of TB to existing medicines. The prevalence of rifampicin resistance for new cases was 1.3\% (95\% CI, 0.8-2.0) and INH resistance among the new cases was 5.5\% (95\% CI, 4.5-6.7). This means that $1.3 \%$ and $5.5 \%$ new TB cases in Kenya receiving 4HREZ/2HR for treatment are actually receiving mono-therapy during the continuation phase and may end up with MDR-TB, which was found in $0.67 \%$ of new cases $(11 / 1,650)$ and $2.1 \%$ amongst previously treated TB cases (4/190). The highest level of mono resistance was for Streptomycin at $6.3 \%$. This could be attributed to previous exposure of Streptomycin particularly in management of brucellosis. Resistance to isoniazid seems to have declined when compared to a national survey carried out in 1974 where $10.1 \%$ had a strain resistant to isoniazid and/or streptomycin, 7.3\% to isoniazid alone, $1.4 \%$ to Streptomycin alone and $1.4 \%$ to both drugs [10]. Decline in isoniazid resistance is likely to be attributed to protection that isoniazid must have received with the introduction of rifampicin in combined therapy. Resistance to Streptomycin similarly seems to have declined from $7.3 \%$ in 1974 . This is also likely to be due to the fact that streptomycin is no longer used in treatment of TB after category two treatment regimes were stopped in Kenya. There was no resistance pattern for rifampicin in 1974 since the drug was not commonly used to treat TB.

These results demonstrate an increase of MDR TB amongst new cases by $24 \%$ to $0.67 \%$ (using 1974 cases as baseline). This increase in resistance within a span of 41 years is a slow but worrisome phenomenon that needs to be urgently addressed by the program as it translates to an equivalent proportion of increase in new MDR TB patients with enormous programmatic consequences. The decline of MDR TB cases from previously treated cases declined in the same period from $8.54 \%$ to $2.1 \%$ possibly due to the lethal impact of HIV where those who were dually infected died before reporting.

Although sampling of patients for previous surveys in Kenya have not included refugee population, higher resistance levels were reported in 2,000 amongst refugee population of Kenya compared to non-refugee population [OR $=3.7 ; 95 \%$ CI 1.42-9.68; $p<0.007$ ] [11]. At that time, $2.9 \%$ of sampled refugee population was found to have MDR TB while the non-refugee population did not report any MDR TB. During this time $1.1 \%$ of the non-refugee population had rifampicin resistance and $2.3 \%$ had isoniazid resistance. Although these figures are higher than what this survey is reporting in 2015, the sample of the non-refugee population in 2,000 was amongst the local natives living and interacting with the refugee population. DNA analysis of resistant strains from the 
non-refugee population was unique and different from those in the refugee camps [11]. The refugee populations at the camps were from Somalia where TB control activities had basically collapsed, an indication that a well-functioning program plays a role in reducing development of drug resistance. In addition, it was found that DNA fingerprints of resistant strains from the non-refugee population were unique and different from those in the refugee camps [11].

It is anticipated that MDR TB may be more common in the urban set up due to the increased risk factors in urban settings [12]. In a study conducted in 2,000 in Nairobi, the capital city of Kenya (a number of refugees diffuse into the local community), the resistance to isoniazid, streptomycin and MDR TB amongst new TB cases was $10.3 \%, 4.3 \%$ and $0.54 \%$ respectively while for previously treated TB patients, the resistance was $18.1 \%$ for isoniazid, $10.5 \%$ for Streptomycin, 9.04\% for rifampicin and 8.4\% MDR TB [13]. These results demonstrate that there exists an increase in MDR TB amongst new cases (by $24 \%$ to $0.67 \%)$.

TB control in Kenya has a long history. Kenya laid down the foundation for TB control in 1956 when the National TB program was started and tasked with providing control activities including diagnosis, treatment and occasional mass campaigns using miniature chest X-rays [14]. Subsequently, the Ministry of Health in 1973, issued a circular that described TB diagnostic procedures, chemotherapy, case-holding, bacteriological monitoring, and recording and reporting systems to be implemented in all districts [15]. Presently, Kenya has developed ambitious targets through the Health policy to eliminate communicate diseases by 2030 and TB is amongst the diseases earmarked for elimination [16]. Development and expanding MDR TB menace threatens this noble goal.

Kenya adopted rifampicin containing regimen for treatment of TB in 1993 when the country introduced fixed dose combination tablets under directly observed treatment-short course (DOTS). By 1997, the whole country had adopted the use of the fixed dose combination given in programmatic conditions. Over the years, favorable treatment outcomes have been achieved and remained consistently over $80 \%$ cure rates. Although observation was initially health facility based (health provider) during the intensive phase, introduction of community Dots meant that the patients were observed taking medication at the convenience of their homes when community dots programs were widely expanded from 2006. In addition, the late entry of rifampicin into the Kenyan market and limited use has preserved the medicine form developing resistance particularly by TB.

This survey was not designed to investigate possible risk factors. With the low numbers of MDR TB found in most surveys, a significant association is rarely found; except for treatment history (a history of being previously treated is always associated with MDR TB). The major risk factor for tuberculosis is HIV. In Kenya, HIV is still a major driver of TB with a national seroprevalence of the general population aged 15-64 standing at 5.6\% [17]. It has also been documented that HIV influences the natural history of $\mathrm{TB}$ and lead to emergence of acquired drug resistance in individual patients during treatment [18]. About $20.6 \%$ of the participants in the survey were dually infected with HIV compared to 36\% for notified TB cases [19]. The low TB and HIV co-infection rate found in this survey compared to the national reported rate could be attributed to early mortality associated with dual combination [20,21] and other unforeseen factors. Many patients who develop MDR TB have undergone treatment with first line medicines and failed. The Kenyan policy prior to introduction of Genexpert as the first line of diagnosis has culture of sputum from patients who fail first line treatment. By the time the results are released from the NTRL, some of these patients, who may have developed drug resistant TB, may have succumbed to the disease. 
It is however interesting to note that diabetes was identified as a risk factor in this study and would require further investigation with a larger representative sample size amongst the diabetes patients. As the country transitions from infectious diseases to non communicable diseases, diabetes is expected to increase and therefore play a big role in TB transmission.

NTM (Non tuberculous TB) is a problem that has existed even before HIV complicated TB control. In 1974 survey in Kenya, $0.25 \%$ of the culture positive specimen who was neither Mycobacteria tuberculosis nor Mycobacteria bovis [10] may have passed as NTM's (Non Tuberculous Mycobacteria). Low levels of (NTM) were identified from culture of submitted specimen. Identification of 10 cases of NTM (Four HIV negative, five HIV positive and one unknown HIV status) has shown that NTM's may be a growing but a neglected problem by the program as there are no guidelines on how to manage these types of patients. The false believe that NTM's may not be causing a health problem need to be revisited in light of the new evidence. NTM's generally present with symptoms similar to TB and may end up being managed as TB especially in places where ZN staining is used to diagnose TB, as NTM's stain like TB. In particular, NTM's are more common in males and smokers. This survey showed that 4 out of $10(40 \%)$ were HIV negative, an indication that its association with HIV is not always true. Programs must make extra efforts to manage these conditions. In addition, more efforts need to be put in place of characterize the common types of NTM's seen.

This survey revealed that more males than females are infected with TB in a ratio of 2:1 [22, 23]. The reasons behind the gender disparity are not clear but a number of hypotheses have been advanced. These include disparities in seeking care and accesses to health care by gender. Several factors including stigma associated with tuberculosis have been advanced. Social factors such as smoking, alcoholism and poor nutrition may also play a part in predisposing men to Tuberculosis [24].

This survey further confirms a known fact that TB disease is prevalent among the economically active age group. In this study, the groups encompass about $79 \%$ of the respondents. This further stresses the need for concerted efforts to combat TB since it has serious economic consequences to the national economy. A good health system should be dynamic enough to provide required health care in order to maintain a vibrant economy.

The literacy levels of the study participants are reflective of the national literacy levels and give clear evidence that TB is wide spread across all educational levels and age ranges.

\section{Conclusions}

Although drug resistance TB is a growing problem in Kenya, resistance to isoniazid and rifampicin (MDR TB) is less than that was previously estimated. The low levels of resistance is attributed to several factors and especially to recent exposure of rifampicin to patients with $\mathrm{TB}$ in Kenya as well as a well-functioning TB control program that has ensured excellent case holding of cases initiated on treatment. However, when translated to actual patients, the increase is worrisome and needs urgent mitigation. There is a need to ensure routine monitoring of drug resistance patterns and ensure that TB control program continues to maintain a robust $\mathrm{TB}$ surveillance system.

\section{Acknowledgements}

Special thanks are made to Centres for Disease Prevention and Control, Kenya, for funding this survey through MoH-CDC Coag. Special consideration is placed on patients whose specimen was collected and made this survey possible.

\section{References}

[1] Zhao, Y., Xu, S., Wang, L., Chin, D. P., Wang, S., Jiang, G., and Pang, Y. 2012. "National Survey of 
Drug-Resistant Tuberculosis in China.” New England Journal of Medicine 366 (23): 2161-70.

[2] Raviglione, M., and Director, G. T. 2013. "Global Strategy and Targets for Tuberculosis Prevention, Care and Control after 2015.”

[3] Mekonnen, F., Tessema, B., Moges, F., Gelaw, A., Eshetie, S., and Kumera, G. 2015. "Multidrug Resistant Tuberculosis: Prevalence and Risk Factors in Districts of Metema and West Armachiho, Northwest Ethiopia.” BMC Infectious Diseases 15 (1): 461.

[4] Spellberg, B., Powers, J. H., Brass, E. P., Miller, L. G., and Edwards, J. E. 2004. "Trends in Antimicrobial Drug Development: Implications for the Future." Clinical Infectious Diseases 38 (9): 1279-86.

[5] Pablos-Méndez, A., Raviglione, M. C., Laszlo, A., Binkin, N., Rieder, H. L., Bustreo, F., and Nunn, P. 1998. "Global Surveillance for Antituberculosis-Drug Resistance, 1994-1997.” New England Journal of Medicine 338 (23): 1641-9.

[6] World Health Organization. 2015. Guidelines for Surveillance of Drug Resistance in Tuberculosis.

[7] Rabna, P., Ramos, J., Ponce, G., Sanca, L., Mané, M., Armada, A., and Colombatti, R. 2015. "Direct Detection by the Xpert MTB/RIF Assay and Characterization of Multi and Poly Drug-Resistant Tuberculosis in Guinea-Bissau, West Africa." Plos One 10 (5): e0127536.

[8] Drobniewski, F. A, Balabanova, Y. M, Ruddy, M. C, Graham, C., Kuznetzov, S. I, Gusarova, G. I., et al. 2008. "Tuberculosis, HIV Seroprevalence and Intravenous and Drug Abuse in Prisoners.” EurRespir J. 26 (2): 298-304.

[9] WHO (World Health Organization). WHO Antimicrobial Resistance: Global Report on Surveillance, April 2014.

[10] African, E. 1978. "Tuberculosis in Kenya: a Second National Sampling Survey of Drug Resistance and Other Factors, and a Comparison with the Prevalence Data from the First National Sampling Survey." Tubercle 59 (3): 155-77.

[11] Githui, W. A., Hawken, M. P., Juma, E. S., Godfrey-Faussett, P., Swai, O. B., Kibuga, D. K., and Drobniewski, F. A. 2000. "Surveillance of Drug-Resistant Tuberculosis and Molecular Evaluation of Transmission of Resistant Strains in Refugee and Non-refugee Populations in North-Eastern Kenya.” The International Journal of Tuberculosis and Lung Disease 4 (10): 947-55.

[12] Almeida, D., Rodrigues, C., Udwadia, Z. F., Lalvani, A.,
Gothi, G. D., Mehta, P., and Mehta, A. 2003. "Incidence of Multidrug-Resistant Tuberculosis in Urban and Rural India and Implications for Prevention.” Clinical Infectious Diseases 36 (12): e152-4.

[13] Ogaro, T. D., Githui, W., Kikuvi, G., Okari, J., Wangui, E., and Asiko, V. 2012. "Anti-tuberculosis Drug Resistance in Nairobi, Kenya.” Afr J. Health 20: 21-7.

[14] MoH, Control of Tuberculosis. Nairobi, Ministry of Health, 1973.

[15] World Health Organization. 2009. “A Brief History of Tuberculosis Control in Kenya.”

[16] Ministry of Health. 2014. Kenya Health Policy 2012-2030. Nairobi: Ministry of Health.

[17] National AIDS and STI Control Program, Ministry of Health, Kenya. September 2013. Kenya AIDS Indicator Survey 2012: Preliminary Report. Nairobi, Kenya.

[18] Small, P. M, Schecter, G. F, Goodman. P. C, Sande, M. A, Chaisson, R. E, and Hopewell, P. C. 1991. "Treatment of Tuberculosis in Patients with Advanced Human Immunodeficiency Virus Infection.” N Engl J. Med. 324: 289-94.

[19] NTLD-P 2016. National Tuberculosis Leprosy and Lung Disease Program, Annual Report 2015. Nairobi: NTLD-P.

[20] Campos, P. E., Suarez, P. G., Sanchez, J., Zavala, D., Arevalo, J., Ticona, E., and Holmes, K. K. 2003. "Multidrug-Resistant Mycobacterium Tuberculosis in HIV-Infected Persons, Peru.” Emerging Infectious Diseases $\quad 9 \quad$ (12): $1571-8$ https://dx.doi.org/10.3201/eid0912.020731.

[21] O’Donnell, M. R., Padayatchi, N., Kvasnovsky, C., Werner, L., Master, I., and Horsburgh, C. 2013. "Treatment Outcomes for Extensively Drug-Resistant Tuberculosis and HIV Co-infection.” Emerging Infectious Diseases $19 \quad$ (3): 416-24. https://dx.doi.org/10.3201/eid1903.120998.

[22] Sitienei, J., Nyambati, V., and Borus, P. 2013. "The Epidemiology of Smear Positive Tuberculosis in Three TB/HIV High Burden Provinces of Kenya [2003-2009].” Epidemiology Research International.

[23] Kipruto, H., Mung'atu, J., Ogila, K., Adem, A., Mwalili, S., Masini, E., and Kibuchi, E. 2015. The Epidemiology of Tuberculosis in Kenya, a High TB/HIV Burden Country [2000-2013].

[24] WHO. 2013. "Guideline: Nutritional Care and Support for Patients with Tuberculosis.” Geneva: World Health Organization. 